\title{
Inimputabilidade: estudo dos internos do Instituto Psiquiátrico Forense Maurício Cardoso
}

\author{
Nonimputability: a study on inmates at Instituto Psiquiátrico Forense Maurício \\ Cardoso
}

\author{
Gabriel José Chittó Gauer ${ }^{1}$, Fernanda Correa Osório² , Alfredo Cataldo Neto ${ }^{3}$, Letícia \\ Teixeira ${ }^{4}$, Mariane Caum${ }^{4}$, Taís Amaral da Costa Souza ${ }^{4}$, Verônica Valle ${ }^{4}$, Vivian Cristófoli ${ }^{4}$
}

\begin{abstract}
${ }^{1}$ Psiquiatra. Professor, Programa de Pós-Graduação: Psicologia, e Programa de Mestrado em Ciências Criminais, Pontifícia Universidade Católica do Rio Grande do Sul (PUCRS), Porto Alegre, RS. ${ }^{2}$ Advogada, Universidade Luterana do Brasil (ULBRA), Canoas, RS. Mestre em Ciências Criminais, PUCRS. Professora, Faculdades Planalto, Centro Universitário La Salle, Canoas, RS. ${ }^{3}$ Psiquiatra. Professor adjunto, Departamento de Psiquiatria e Medicina Legal, Faculdade de Medicina (FAMED), PUCRS. Programa de Mestrado em Ciências Criminais, PUCRS. ${ }^{4}$ Acadêmicas de Medicina, FAMED, PUCRS.

Trabalho baseado na dissertação de mestrado intitulada "Inimputabilidade: estudo dos internos de um instituto psiquiátrico forense”, defendida em 2006 no Programa de Mestrado em Ciências Criminais da Faculdade de Direito da Pontifícia Universidade Católica do Rio Grande do Sul (PUCRS), Porto Alegre, RS.
\end{abstract}

\section{Resumo}

Introdução: A pesquisa traçou um perfil dos internos inimputáveis do Instituto Psiquiátrico Forense Maurício Cardoso.

Métodos: Durante o período de abril a agosto de 2005, foram selecionados 617 sujeitos para inclusão no estudo.

Resultados: A análise dos dados revelou que os internos apresentam as seguintes características: idade média de 43,22 anos, sexo masculino (91,3\%), profissão definida (73,2\%), primeiro grau completo $(74,6 \%)$, sem companheiro(a) $(83,2 \%)$, crime contra a pessoa como delito gerador da inimputabilidade (62,1\%), com antecedentes criminais (58,3\%), prazo mínimo fixado na sentença de 1,76 anos, cumprindo medida de segurança detentiva (91,4\%), média do tempo de internação de 9,33 anos, sob o regime de alta progressiva (81,5\%), com internação prévia em outras instituições psiquiátricas (55,2\%), sem cumprimento anterior de medida de segurança (83,3\%), com o diagnóstico de esquizofrenia e outros transtornos psicóticos (61,4\%). Os cruzamentos de variáveis que tiveram relevância estatística foram: dos internos com internação prévia, 61,9\% possuíam antecedentes criminais, e quanto maior o nível de escolaridade do interno, menor o índice de reincidência. Evidenciou-se uma preponderância de internos com antecedentes criminais em pacientes com transtornos relacionados a substâncias (56,5\%) e transtorno de personalidade (59,7\%). Verificou-se que $60,8 \%$ daqueles que cometeram crimes contra a pessoa possuíam diagnóstico de transtornos relacionados ao álcool, estabelecendo uma relação entre criminalidade violenta e os transtornos de uso de substâncias.

Conclusões: A partir da análise dos prontuários dos 617 internos do Instituto Psiquiátrico Forense Maurício Cardoso, o presente estudo procurou apresentar um mapa dos indivíduos considerados inimputáveis no Rio Grande do Sul.

Descritores: Inimputabilidade, medida de segurança, violência.

\begin{abstract}
Introduction: This study analyzed the profile of nonimputable inmates at Instituto Psiquiátrico Forense Maurício Cardoso. Methods: Between April and August 2005, a total of 617 patients were selected for inclusion in the study.

Results: Data analysis revealed that the inmates presented the following characteristics: mean age of 43.22 years, male (91.3\%), defined profession (73.2\%), completed elementary school $(74.6 \%)$; without a partner $(83.2 \%)$, crime against a person was the felony that generated nonimputability (62.1\%), criminal records $(58.3 \%)$, minimal sentence time was 1.76 years, serving it in criminal commitment (91.4\%), mean time in prison of 9.33 years, under regime of progressive release (81.5\%), previous
\end{abstract}

Gabriel J. Chittó Gauer, Av. Cel. Lucas de Oliveira, 576/501, Mont’Serrat, CEP 90440-010, Porto Alegre, RS. Tel.: (51) 3320.3500, Ramal 7745, Fax: (51) 3028.9090, 3333.2628. E-mail: ggauer@pucrs.br 
hospitalization in other psychiatric institutions (55.2\%), no previous probation measure (83.3\%), diagnosis of schizophrenia and other psychotic disorders (61.4\%). The following variables were statistically significant: among inmates with previous hospitalization, 61.9\% had criminal history; and the higher the inmates' schooling level, the lower the level of criminal relapse. Results showed a predominance of inmates with criminal records in patients with disorders related to substances (56.5\%) and personality disorder (59.7\%). It was also verified that $60.8 \%$ of those who committed crimes against a person were alcohol addicts, establishing a relation between violent criminality and disorders caused by substance misuse.

Conclusions: Based on the analysis of the medical records of 617 inmates at Instituto Psiquiátrico Forense Maurício Cardoso, this study aimed at presenting a map of individuals considered nonimputable in Rio Grande do Sul.

Keywords: Nonimputability, criminal commitment, violence.

\section{Introdução}

O presente estudo tem como objetivo traçar um perfil dos indivíduos considerados inimputáveis, agentes sem capacidade de censurabilidade, do Instituto Psiquiátrico Forense Maurício Cardoso (IPFMC), localizado na cidade de Porto Alegre, única instituição destinada a esse fim no estado do Rio Grande do Sul.

Dá-se o nome de capacidade de imputação jurídica ao estado psicológico que se fundamenta no entendimento que o indivíduo tem sobre o caráter criminoso do fato e na aptidão de determinar-se de acordo com esse entendimento. Essa capacidade pode ser total, parcial ou nula. Quando a capacidade de imputação for nula, isso quer dizer que o agente era, à época do delito, totalmente incapaz de entender o caráter criminoso do fato ou totalmente incapaz de determinar-se de acordo com esse entendimento. Assim sendo, o delito por ele praticado lhe é inimputável, e ele, agente, poderá ser julgado penalmente irresponsável pelo que fez. Dessa forma, a capacidade de imputação jurídica depende da faculdade do indivíduo de entender e de determinar-se. A faculdade de entender está baseada na possibilidade que o agente tem de conhecer a natureza, as condições e as conseqüências do ato. Implica o conhecimento da penalidade, da organização legal, das conseqüências sociais e supõe certo grau de experiência, de maturidade, de educação, de inteligência, de lucidez, de orientação e de memória. A faculdade de se determinar baseia-se na capacidade de escolher entre praticar ou não o ato, o que requer serenidade, reflexão e distância de qualquer condição patológica que possa escravizar a vontade do indivíduo, impulsionando-o para o ato ${ }^{1,2}$.

A lei penal brasileira exige, para responsabilizar o autor de um crime, não mais do que a consciência do caráter delituoso do fato e a liberdade de escolha entre praticá-lo ou não. Reza o artigo 26 do Código Penal que é isento de pena o agente que, por doença mental ou desenvolvimento mental incompleto ou retardado, era, ao tempo da ação ou omissão, inteiramente incapaz de entender o caráter ilícito do fato ou de determinar-se de acordo com esse entendimento ${ }^{3}$.

O IPFMC é um estabelecimento médico penal da rede penitenciária do Governo do Estado do Rio Grande do Sul vinculado à Secretaria dos Serviços
Penitenciários. Destinado à internação e à reabilitação dos infratores doentes mentais submetidos à medida de segurança e de sentenciados que foram acometidos de doença mental. Também o instituto tem a função de, durante o processo judicial, realizar exames e perícias para a verificação de responsabilidade e de periculosidade penal.

A presente pesquisa busca identificar as principais características dos internos que cumprem medida de segurança no IPFMC.

Dessa forma, a pesquisa, na tentativa de auxiliar a traçar o mapa da inimputabilidade no Rio Grande do Sul, contribui para o debate sobre a temática que envolve medida de segurança, doença mental e criminalidade.

\section{Método}

\section{Amostra}

O estudo foi realizado no IPFMC. A população em estudo foi formada por todos os pacientes inimputáveis no período de abril a agosto de 2005. Estava em tratamento um total de 617 pacientes inimputáveis na época de referência. Foram excluídos do estudo aqueles que aguardavam julgamento, os declarados semi-imputáveis e os que cumprem medida de segurança por superveniência de doença mental.

Os dados foram coletados dos prontuários judiciais arquivados no IPFMC através de um instrumento específico. Também foram realizadas as análises das sentenças judiciais, dos laudos de avaliação de responsabilidade penal, dos laudos de verificação de cessação da periculosidade e outros documentos. Os dados sociodemográficos, como a idade, o grau de instrução e o estado civil, foram coletados com base na situação atual do interno, não no momento da prática do fato criminoso ou no momento da declaração de inimputabilidade.

\section{Tratamento dos dados}

Os resultados foram digitados em um banco de dados no pacote estatístico SPSS 10.0 (SPSS Inc., Chicago, 2000), e foi realizada, inicialmente, uma análise 
descritiva univariada dos mesmos, para cálculo das freqüências, médias e desvios padrões. Num segundo momento, foi realizada uma análise bivariada. Para relacionar as variáveis independentes, tais como sexo, faixa etária e nível socioeconômico, com as variáveis dependentes, como tipo de delito, antecedentes criminais, tempo de internação e outras, foi aplicado um teste de associação entre variáveis (qui-quadrado de independência). A existência ou não de associações estatisticamente significativas entre as médias, no caso das variáveis quantitativas, foi verificada através do teste $t$ de Student, para amostras independentes. Foi considerado um nível de significância de p $\leq$ 0,05.

\section{Aspectos éticos/legais}

O projeto de pesquisa foi submetido e aprovado pelo diretor do IPFMC, pela juíza da Vara de Execuções de Penas e Medidas de Segurança e pelo Comitê de Ética em Pesquisa da PUCRS.

\section{Resultados}

\section{Características gerais da amostra}

De acordo com os prontuários analisados, os internos inimputáveis que se encontravam no IPFMC no período de abril a agosto de 2005 apresentam as seguintes características: idade média de 43,22 anos de idade, com uma predominância masculina $(91,3 \%)$, sendo que a maioria possui profissão definida (73,2\%). Quanto ao grau de escolaridade, encontramos as seguintes porcentagens: $74,6 \%$ haviam cursado o primeiro grau; $16,4 \%$ eram analfabetos; 7,3\% cursaram o segundo grau; e 1,6\% freqüentaram curso superior. A grande maioria não possuía companheiro(a) no momento da coleta dos dados (83,2\%). O delito gerador da inimputabilidade mais prevalente entre os internos foi o crime contra a pessoa (62,1\%), seguido do crime contra o patrimônio (20,6\%) e crime contra os costumes (16,2\%). Em menor porcentagem, aparecem os crimes contra a incolumidade pública $(3,4 \%)$, crimes constantes na Lei de Tóxicos (2,6\%), crimes contra a administração pública $(2,3 \%)$, crimes contra os sentimentos religiosos e respeito aos mortos $(0,6 \%)$, contravenções penais $(0,5 \%)$, crimes contra a fé pública $(0,3 \%)$ e crimes constantes na Lei de Tortura $(0,3 \%)$. Os resultados apontaram uma prevalência de $58,3 \%$ de internos com antecedentes criminais. Quanto ao prazo mínimo fixado para a medida de segurança, verificou-se que a média é de 1,76 anos. Observou-se uma predominância significativa da medida de segurança detentiva (91,4\%). A média do tempo de internação da amostra é de 9,33 anos, sendo que metade dos internos estava na instituição há mais de 7 anos, e o maior tempo de internação encontrado foi de 41 anos. Quanto à situação do interno dentro da instituição, verificou-se que a maioria encontra-se sob o regime de alta progressiva (81,5\%). Quanto à existência de internação prévia em instituição psiquiátrica, verificou-se uma prevalência ligeiramente maior de internos com internação (55,2\%). A grande maioria dos internos pesquisados não havia cumprido medida de segurança anteriormente (83,3\%). Os diagnósticos mais freqüentemente encontrados foram a esquizofrenia e outros transtornos psicóticos (61,4\%), seguido de transtornos relacionados a substâncias (27,5\%), retardo mental e transtorno de comunicação (20,5\%), transtorno de personalidade (12,4\%), transtornos de humor (10\%), transtornos mentais causados por uma condição médica geral (3,4\%), transtornos sexuais (3,1\%), delirium e demência (1,1\%), transtornos de ansiedade $(0,3 \%)$, transtornos dissociativos $(0,2 \%)$, transtornos do sono $(0,2 \%)$ e transtorno de controle dos impulsos $(0,2 \%)$.

\section{Associações}

\section{Associações não-significativas}

Foi verificado que não existem associações estatisticamente significativas entre as seguintes variáveis: sexo e faixa etária ( $p=0,638)$, sexo e grau de instrução ( $p$ $=0,241)$, sexo e existência de profissão definida $(p=0,192)$, sexo e estado civil $(p=0,928)$, sexo e prazo mínimo de internação fixado na sentença $(p=0,191)$, sexo e tempo de internação ( $p=0,741)$, sexo e tipo de medida de segurança $(p=0,198)$, e sexo e internação em outras instituições psiquiátricas $(\mathrm{p}=0,504)$.

\section{Sexo e delito gerador da inimputabilidade}

$\mathrm{Na}$ análise do sexo e delito gerador da inimputabilidade, verifica-se uma preponderância de crimes cometidos contra a pessoa, tanto no sexo masculino como no feminino. Entretanto, entre as mulheres, a porcentagem chega a $84,6 \%$, enquanto que, entre os homens, totalizam 59,9\% dos casos. A Tabela 1 demonstra a distribuição dos internos por sexo e delito gerador da inimputabilidade.

\section{Sexo e existência de antecedentes criminais}

O cruzamento das variáveis sexo e antecedentes criminais demonstra uma associação estatisticamente significante entre as variáveis $(\mathrm{p}<0,001)$. Verificou-se que, entre as mulheres, $86,5 \%$ não possuem e 13,5\% possuem antecedentes criminais. Entre os homens, 44,5\% possuem e $55,5 \%$ não possuem antecedentes criminais.

\section{Sexo e diagnóstico}

Associando-se essas duas variáveis, verifica-se uma maior prevalência do diagnóstico de esquizofrenia e outros transtornos psicóticos, tanto no sexo masculino $(61,4 \%)$ como no sexo feminino $(59,6 \%)$. Verifica-se 
Tabela 1 - Distribuição das variáveis no grupo dos internos, por regime atual e delito gerador

\begin{tabular}{|c|c|c|c|c|c|c|c|c|}
\hline \multirow{3}{*}{ Delito gerador } & \multicolumn{8}{|c|}{ Regime atual } \\
\hline & \multicolumn{2}{|c|}{ Unidade fechada } & \multicolumn{2}{|c|}{ Unidade aberta } & \multicolumn{2}{|c|}{ Alta progressiva } & \multicolumn{2}{|c|}{ Total } \\
\hline & $\mathbf{n}$ & $\%$ & $\mathbf{n}$ & $\%$ & $\mathbf{n}$ & $\%$ & $\mathbf{n}$ & $\%$ \\
\hline Crimes contra a pessoa & 34 & 60,7 & 26 & 44,8 & 321 & 64,3 & 381 & 62,2 \\
\hline Crimes contra o patrimônio & 13 & 23,2 & 15 & 25,9 & 97 & 19,4 & 125 & 20,4 \\
\hline Crimes contra os costumes & 10 & 17,9 & 12 & 20,7 & 78 & 15,6 & 100 & 16,3 \\
\hline Crimes contra a incolumidade pública & 2 & 3,6 & 4 & 6,9 & 15 & 3,0 & 21 & 3,4 \\
\hline Lei de tóxicos & 2 & 3,6 & 1 & 1,7 & 13 & 2,6 & 16 & 2,6 \\
\hline Crimes contra a administração pública & 0 & 0,0 & 1 & 1,7 & 13 & 2,6 & 14 & 2,3 \\
\hline $\begin{array}{l}\text { Crimes contra o sentimento religioso e } \\
\text { contra o respeito aos mortos }\end{array}$ & 0 & 0,0 & 0 & 0,0 & 4 & 0,8 & 4 & 0,7 \\
\hline Contravenções penais & 0 & 0,0 & 1 & 1,7 & 2 & 0,4 & 3 & 0,5 \\
\hline Tortura & 0 & 0,0 & 0 & 0,0 & 2 & 0,4 & 2 & 0,3 \\
\hline Crimes contra a fé pública & 0 & 0,0 & 0 & 0,0 & 2 & 0,4 & 2 & 0,3 \\
\hline Total & 56 & 109,0 & 58 & 103,4 & 499 & 109,5 & 613 & 109,0 \\
\hline
\end{tabular}

também uma maior prevalência do diagnóstico de transtornos relacionados a substâncias nos homens $(29,2 \%)$ em relação às mulheres $(9,6 \%)$. Nota-se, ainda, um maior número de casos de diagnóstico de transtornos de humor no sexo feminino (19,2\%) em relação ao sexo masculino (9,2\%).

\section{Internação em outras instituições psiquiátricas e antecedentes criminais}

Na associação entre essas duas variáveis, observase que, dos internos que possuíam antecedentes criminais, 61,9\% haviam e 38,1\% não haviam sido internados em outras instituições psiquiatras anteriormente. Entre os internos que não possuíam antecedentes criminais, verifica-se que $50,6 \%$ haviam sido internados em outras instituições, e 49,4\% não. Foi verificada a existência de uma associação estatisticamente significativa entre a existência de antecedentes criminais e a internação em outras instituições $(p=0,05)$. A Figura 1 demonstra as associações entre as variáveis antecedentes criminais e internação em outras instituições.

\section{Transtornos relacionados ao álcool, cannabis e cocaína e delito gerador da inimputabilidade}

Ao relacionar essas variáveis, observa-se que $60,8 \%$ dos internos que cometeram algum dos delitos contra a pessoa possuíam um transtorno relacionado ao álcool; 30,4\% possuíam um transtorno relacionado à cocaína; e $26,5 \%$ possuíam transtorno relacionado à cannabis. Nos crimes contra o patrimônio, verifica-se que 52,2\% dos internos possuíam um transtorno relacionado à cocaína; $47,1 \%$, um transtorno relacionado à cannabis; e 20,8\%, um transtorno relacionado ao álcool.

Nos crimes contra os costumes, observa-se que $13,3 \%$ dos internos possuíam um transtorno relacionado ao álcool, e 11,8\% possuíam um transtorno relacionado à cannabis.

Nos crimes tipificados pela Lei de Tóxicos, verificase que $23,5 \%$ dos internos possuíam um transtorno relacionado à cannabis; $21,7 \%$ dos internos possuíam um transtorno relacionado à cocaína; e 4,2\% dos internos possuíam um transtorno relacionado ao álcool.

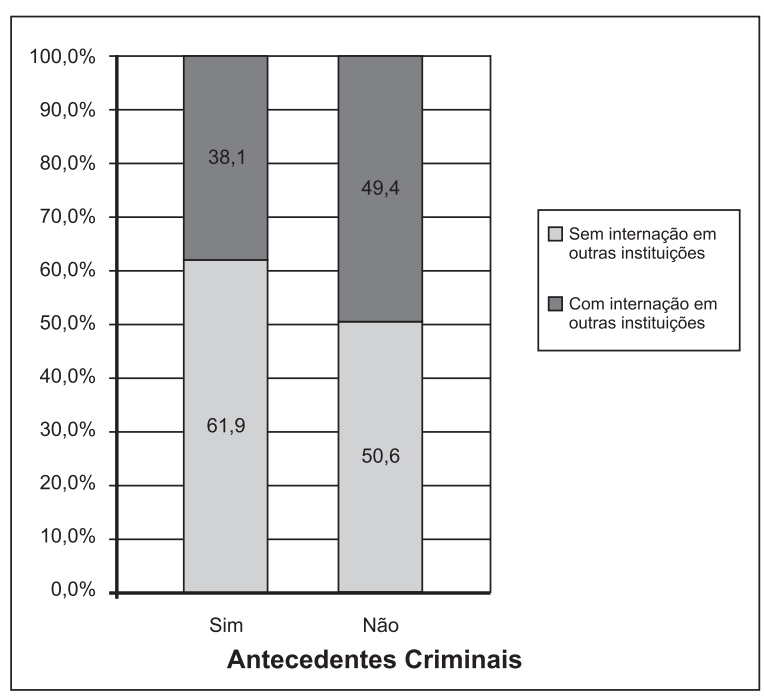

Figura 1 - Internação em outras instituições e antecedentes criminais 


\section{Grau de instrução e existência de antecedentes criminais}

Na associação das variáveis grau de instrução e existência de antecedentes criminais, observa-se que: 49\% dos analfabetos possuíam antecedentes criminais, e 51\% não possuíam; naqueles com o primeiro grau de instrução, 41,9\% possuíam antecedentes criminais, e $58,1 \%$ não; naqueles com o segundo grau de instrução, 33,3\% possuíam antecedentes criminais, e 66,7\% não; e entre os internos com grau superior de instrução, nenhum dos 10 (100\%) possuía antecedentes criminais. Dessa forma, verifica-se que há uma associação estatisticamente relevante entre as variáveis grau de instrução e existência de antecedentes criminais ( $\mathrm{p}=$ 0,014).

\section{Diagnóstico e existência de antecedentes criminais}

No cruzamento entre as variáveis internação prévia em outras instituições psiquiátricas e antecedentes criminais, observa-se uma associação estatisticamente significativa entre as duas variáveis $(p=0,005)$. Dentre os internos que possuíam internação prévia, 61,9\% possuíam e 50,6\% não possuíam antecedentes criminais. Dentre os internos que não possuíam internação em outras instituições, 38,1\% possuíam e 49,4\% não possuíam antecedentes criminais. Infere-se que aqueles internos que possuíam antecedentes criminais, ou seja, um histórico de comportamento violento, já haviam recebido tratamento psiquiátrico anterior.

No cruzamento entre o diagnóstico do interno e a existência de antecedentes criminais, verifica-se que $61,1 \%$ dos internos com o diagnóstico de esquizofrenia e outros transtornos psicóticos não possuíam antecedentes delitivos, e naqueles com transtornos relacionados a substâncias, 56,5\% possuíam. Os internos com diagnóstico de retardo mental e transtorno de comunicação apresentaram um percentual de 51,1\% de antecedentes criminais, e 59,7\% dos internos com o diagnóstico de transtorno de personalidade possuíam antecedentes criminais. Dos 62 casos diagnosticados com transtornos de humor, 56,5\% não possuíam antecedentes delitivos, enquanto que 43,5 possuíam. Dentre os pacientes com o diagnóstico de transtornos mentais causados por uma condição médica geral, verifica-se que 61,9\% não apresentavam antecedentes delitivos, e 38,1\% apresentavam. Dos 19 casos diagnosticados de transtornos sexuais e parafilias, 63,2\% não apresentavam antecedentes delitivos, e 36,8\% apresentavam. A Tabela 2 demonstra a associação entre o diagnóstico e a existência de antecedentes criminais.

Tabela 2 - Distribuição dos internos por diagnóstico e existência de antecedentes criminais (commitment)

\begin{tabular}{lcccccc}
\hline & \multicolumn{3}{c}{ Antecedentes criminais } \\
\cline { 2 - 7 } Diagnóstico & \multicolumn{2}{c}{ Sim } & \multicolumn{2}{c}{ Não } & \multicolumn{2}{c}{ Total } \\
\cline { 2 - 7 } & $\mathbf{n}$ & $\mathbf{\%}$ & $\mathbf{n}$ & $\mathbf{\%}$ & $\mathbf{n}$ & $\mathbf{\%}$ \\
\hline Esquizofrenia e outros transtornos psicóticos & 148 & 38,9 & 232 & 61,1 & 380 & 100,0 \\
Transtornos relacionados a substâncias & 96 & 56,5 & 74 & 43,5 & 170 & 100,0 \\
Retardo mental/transtorno de comunicação & 61 & 48,4 & 65 & 51,6 & 126 & 100,0 \\
Transtornos de personalidade & 46 & 59,7 & 31 & 40,3 & 77 & 100,0 \\
Transtornos do humor & 27 & 43,5 & 35 & 56,5 & 62 & 100,0 \\
Transtornos mentais causados por uma & 8 & 38,1 & 13 & 61,9 & 21 & 100,0 \\
condição médica geral & 7 & 36,8 & 12 & 63,2 & 19 & 100,0 \\
Transtornos sexuais/parafilias & 0 & 0,0 & 7 & 100,0 & 7 & 100,0 \\
Delirium/demência & 0 & 0,0 & 2 & 100,0 & 2 & 100,0 \\
Transtornos de ansiedade & 1 & 100,0 & 0 & 0,0 & 1 & 100,0 \\
Transtornos do controle dos impulsos & 0 & 0,0 & 1 & 100,0 & 1 & 100,0 \\
Transtornos do sono & 0 & 0,0 & 1 & 100,0 & 1 & 100,0 \\
Transtornos dissociativos & 258 & 41,7 & 360 & 58,3 & 618 & 100,0 \\
Total & & & & &
\end{tabular}




\section{Discussão}

De acordo com os resultados, observou-se que os inimputáveis do IPFMC possuem a predominância das seguintes características: sexo masculino, idade média de 43,22 anos, profissão definida, primeiro grau completo, sem companheiro(a), crime contra a pessoa como gerador da inimputabilidade, antecedentes criminais, prazo mínimo fixado na sentença de 1,76 anos, cumprindo medida de segurança detentiva, internado há 9,33 anos, sob o regime de alta progressiva, com internação prévia em outras instituições, não tendo cumprido medida de segurança anteriormente e com o diagnóstico de esquizofrenia e outros transtornos psicóticos.

No que se refere ao sexo dos internos, verificouse a preponderância do sexo masculino (91,6\%), explicada na literatura como o resultado dos fatores biológicos, sociais e culturais que levam os homens a adotarem comportamento de maior risco e, por vezes, mais agressivos do que as mulheres ${ }^{4,5}$.

Em relação à média de idade e ao sexo dos internos, verificou-se que a faixa etária prevalente, tanto dos internos masculinos quanto das internas femininas, é a de 40 a 49 anos (28\% dos internos e $36,5 \%$ das internas). Provavelmente, o agravamento do transtorno, com o passar dos anos, e os múltiplos episódios produtivos, associados à piora do quadro clínico, seja um dos fatores relacionados ao cometimento do delito em idade mais tardia do que a população carcerária que tem o início mais precoce de práticas de delitos ${ }^{4,5}$.

$\mathrm{Na}$ associação entre as variáveis sexo e grau de instrução, observou-se que o primeiro grau completo é o mais prevalente, tanto para os internos masculinos (74,1\%) como para os femininos (78,8\%). O baixo grau de instrução dos internos do IPFMC pode ser explicado pelas suas condições de pobreza, agravadas pelo difícil acesso a tratamentos especializados e pela limitada possibilidade para a aprendizagem devido ao transtorno mental.

Verificou-se que há uma associação estatisticamente relevante entre as variáveis grau de instrução e existência de antecedentes criminais ( $\mathrm{p}=$ 0,014). Isto é, quanto maior o nível de escolaridade dos internos, menor são os índices de reincidência criminal: dentre os analfabetos, $49 \%$; naqueles com o primeiro grau de instrução, 41,9\%; naqueles com o segundo grau de instrução, 33,3\%; e nos com instrução superior, nenhum dos 10 internos analisados possuía antecedentes criminais.

Apesar da pequena amostra de sujeitos com nível superior, uma suposição que pode ser feita é de que quanto maior o nível de escolaridade, maior a condição socioeconômica do interno e, conseqüentemente, maiores as condições de acesso a tratamento psiquiátrico especializado, o que diminui as chances de envolvimento em conflitos e práticas delituosas.

$\mathrm{Na}$ associação entre os sexo e o estado civil, verifica-se que tanto o interno masculino (83,3\%) como o interno feminino $(82,7)$ não possuem companheiro(a). Verificou-se uma maior incidência de internos sem companheiro(a) $(83,2 \%)$ sobre os com companheiro(a) (16,8\%). Podemos supor que, no caso do infrator doente mental, a doença somada à violência dificulta ainda mais a possibilidade de estabelecer vínculos permanentes.

O cruzamento das variáveis sexo e antecedentes criminais demonstra uma associação estatisticamente significante entre as variáveis $(\mathrm{p}<0,001)$. Observa-se que a proporção de homens com antecedentes criminais (44,5\%) é maior do que a de mulheres na mesma condição (13,5\%), comprovando, então, a maior incidência de um passado violento entre os homens, como se pode observar na preponderância do sexo masculino na população carcerária. Os fatores biopsicossociais que contribuem para uma maior prevalência de comportamento violento nos homens em relação às mulheres têm sido amplamente estudados ${ }^{6-8}$.

Relacionando as variáveis sexo e internação prévia em outras instituições psiquiátricas, observouse que $59,6 \%$ das mulheres e $54,8 \%$ dos homens internos no IPFMC já haviam sido internados em instituições psiquiátricas. Não foram encontradas associações estatisticamente significantes entre o sexo e a ocorrência de internação em outras instituições ( $\mathrm{p}=0,504)$; a maioria dos internos, homens ou mulheres, possuía um histórico prévio de internação psiquiátrica, o que está de acordo com a idéia de que o comportamento delituoso está relacionado à cronicidade do quadro clínico e à presença de surtos anteriores.

No cruzamento entre as variáveis diagnóstico e internação prévia em instituições psiquiátricas, observou-se que: dentre os internos com diagnóstico de esquizofrenia, a maioria (60,9\%) já possuía internação prévia; o mesmo foi observado em internos com diagnóstico de transtornos relacionados a substâncias (54,8\%), retardo mental e transtornos de comunicação (51,6\%), transtornos de humor (62,9\%) e transtornos mentais causados por uma condição médica geral (52,4\%). Uma vez mais, uma das explicações para a existência de internação prévia é a gravidade desses transtornos mentais, que se caracterizam, na sua grande maioria, por uma desordem profunda nos processos psíquicos, com graves sintomas mórbidos, necessitando de abordagem terapêutica.

Entretanto, verificou-se que a existência de internação psiquiátrica prévia não foi observada nos 
diagnósticos de transtornos de personalidade e transtornos sexuais. Nos transtornos de personalidade, observa-se que $64,5 \%$ dos internos, ou seja, a maioria, não possuíam internação anterior. Nos transtornos sexuais, observa-se que $89,5 \%$ dos internos também não possuíam internação anterior. Porém, sabe-se que pacientes com transtornos de personalidade, em especial aqueles com transtorno de personalidade anti-social, com grande freqüência apresentam problemas precoces com a justiça, e suas dificuldades são abordadas pelo próprio judiciário ou sistema penal e não pelos profissionais da saúde.

Dentre os internos que possuíam internação prévia, $61,9 \%$ possuíam antecedentes criminais, e 50,6\% não possuíam antecedentes. Dentre os internos que não possuíam internação em outras instituições, 38,1\% possuíam antecedentes criminais, e 49,4\% não possuíam antecedentes delitivos. Infere-se que aqueles internos que possuíam antecedentes criminais, ou seja, um histórico de comportamento violento, já haviam recebido tratamento psiquiátrico anterior.

$\mathrm{Na}$ análise do sexo e delito gerador da inimputabilidade, verificou-se uma preponderância de crimes cometidos contra a pessoa, tanto no sexo masculino como no feminino. Entretanto, entre as mulheres, a porcentagem chega a $84,6 \%$, enquanto que, entre os homens, os crimes contra a pessoa como delito gerador da inimputabilidade totalizam $59,9 \%$ dos casos.

Associando-se as variáveis sexo e diagnóstico, verifica-se uma maior prevalência do diagnóstico de esquizofrenia e outros transtornos psicóticos, tanto no sexo masculino $(61,4 \%)$ como no sexo feminino (59,6\%). Em $61,1 \%$ dos internos com o diagnóstico de esquizofrenia, ou seja, a maioria, não há antecedentes delitivos, enquanto que, nos transtornos relacionados a substâncias, 56,5\% apresentavam antecedentes criminais, e 59,7\% dos internos com o diagnóstico de transtornos de personalidade também apresentavam histórico de comportamento violento.

$\mathrm{Na}$ análise do cumprimento anterior de medida de segurança pelos internos do IPFMC, observou-se que $62,5 \%$ dos internos com o diagnóstico de esquizofrenia não haviam cumprido medida de segurança anterior. Já os internos com o diagnóstico de transtornos relacionados a substâncias e transtornos de personalidade apresentavam maiores índices de cumprimento anterior de medida de segurança; com transtornos de personalidade, 25,2\% já haviam cumprido medida de segurança, enquanto que $9,9 \%$ não; com transtornos relacionados a substâncias, 34\% já haviam cumprido medida de segurança, enquanto que 26,3\% não. Dessa forma, em termos longitudinais, os transtornos de personalidade e transtornos relacionados a substâncias possuem uma maior interligação com a criminalidade e violência se comparados à esquizofrenia. Demonstrou-se, ainda, uma maior prevalência do diagnóstico de transtornos relacionados a substâncias nos homens (29,2\%) em relação às mulheres $(9,6 \%)$.

Na análise dos transtornos relacionados ao álcool, cannabis e cocaína e delito gerador da inimputabilidade, foi demonstrada a existência de uma relação entre a criminalidade violenta e os transtornos relacionados ao álcool. Ao relacionar essas variáveis, observou-se que $60,8 \%$ dos internos que cometeram delitos contra a pessoa possuíam um transtorno relacionado ao álcool; 30,4\% possuíam transtorno relacionado à cocaína; e $26,5 \%$ possuíam transtorno relacionado à cannabis.

No tocante à situação do interno na instituição, observou-se que $81,5 \%$ ou 501 internos encontram-se sob alta progressiva; o restante dos pacientes, que não se encontra sob esse regime, está na unidade fechada $(9,1 \%$ ou 56 internos) e na unidade aberta (9,4\% ou 58 internos).

Quanto ao prazo mínimo de cumprimento da medida de segurança, verificou-se que, em $56,2 \%$ dos casos analisados, não ultrapassam 2 anos, e, em 40,7\%, esse prazo varia de 2 a 4 anos.

O sexo do doente mental infrator não influenciou na fixação do prazo mínimo de cumprimento da medida de segurança. Ressalta-se que, durante a pesquisa nos prontuários dos internos do IPFMC, foram encontrados prazos superiores ao mínimo legal, pelo fato de serem anteriores à Reforma da Parte Geral do Código Penal de 1984, que limitou o prazo mínimo de internação.

Quanto ao tipo de medida de segurança, 91,4\% ou 562 internos cumprem medida de segurança detentiva, e $8,6 \%$ ou 53 internos, dos 615 casos analisados, cumprem medida de segurança restritiva.

\section{Conclusão}

A partir da análise dos prontuários dos 617 internos do IPFMC, instituição destinada à internação e reabilitação do doente mental infrator, o presente estudo objetivou traçar um mapa dos indivíduos considerados inimputáveis no Rio Grande do Sul.

Um dos fatos verificados no presente estudo é a relação entre a transgressão cometida e a existência de um transtorno mental grave, de evolução crônica, num indivíduo com episódios prévios e de um baixo nível socioeconômico, o que evidencia que a falta de políticas públicas e de apoio da comunidade em geral é um dos grandes fatores que contribuem para que o comportamento violento ocorra no paciente com transtorno mental. O objetivo do presente trabalho não é aprofundar o estudo comparativo da nossa realidade com a de outros países, e sim fornecer dados do nosso 
meio. Entretanto, tais fatos, com certeza, não são um privilégio do paciente psiquiátrico brasileiro, e relatos de situações onde características semelhantes são encontradas podem ser verificados na literatura internacional $^{9}$. Portanto, os dados aqui apresentados revelam a importância do suporte, tanto em nível de atenção na área da saúde como social, para pessoas com transtornos mentais.

Acredita-se que, com esses dados, possamos contribuir na formulação de políticas apropriadas para o aparelhamento dos serviços mentais, na medida em que o perfil dos pacientes internados é analisado. Este estudo foi realizado nessa perspectiva da necessidade de investigações na área de interface da saúde mental e seus transtornos com a violência.

\section{Referências}

1. Cataldo Neto A, Gauer GJC, Osório FC, Teixeira L, Caum M, Valle V. Inimputabilidade e doença mental. In: Gauer RMC, ed. Sistema penal e violência. Rio de Janeiro: Lúmen Júris; 2007. p. 157-63.

2. Breier A, Paz RA, Gauer GC. Imputabilidade: uma análise crítica. In: Gauer GC, ed. Agressividade uma leitura biopsicossocial. Curitiba: Juruá; 2001. p. 149-62.

3. Hungria N, Flagoso HC. Comentários ao código penal. Rio de Janeiro: Forense; 1983.

4. Black DW. Bad boys, bad men: confronting antisocial personality disorder. New York: Oxford University; 1999.

5. Niehoff D. The biology of violence. New York: Free; 1999.

6. Gauer GJC, Guilhermano TF. Fatores biológicos associados à conduta agressiva. In: Gauer GC, ed. Agressividade uma leitura biopsicossocial. Curitiba: Juruá; 2001. p. 11-37.

7. Stout M. The sociopath next door. New York: Bradway; 2005.

8. Gauer GJC, Cataldo Neto A. Transtorno de personalidade antisocial. In: Cataldo Neto A, Gauer GJC, Furtado NR, eds. Psiquiatria para o estudante de medicina. Porto Alegre: EDIPUCRS; 2003. p. 595-608.

9. O’Malley S. Are you there alone? New York: Simon \& Schuster; 2004 